\title{
The effect of irradiation on a medulloblastoma
}

\author{
D. R. OPPENHEIMER
}

From the Department of Neuropathology, Radcliffe Infirmary, Oxford

The radiosensitivity of medulloblastomas has been recognized for the past 40 years (Bailey, Sosman, and van Dessel, 1928). There have been descriptions of histological changes in the tumour tissue after irradiation (for example, Bailey, 1930; Alpers and Pancoast, 1933; Tarlov, 1937; Frazier, Alpers, Pendergrass, and Chamberlin, 1937) which have emphasized the destruction of tumour cells and subsequent intense fibrous scarring in and around the tumour. The present paper calls attention to a peculiar transformation in the tumour tissue, occurring some 10 days after irradiation, which might in some circumstances lead to diagnostic difficulties. In the case to be described the irradiated tissue could be compared, post-mortem, with unirradiated tumour, as well as with previous biopsy specimens; in other words, the case provided a kind of controlled experiment on the effects of radiation.

\section{CASE HISTORY}

The patient, I.T., was a boy aged 10 when he presented with a few weeks' history of nausea, vomiting, dizziness, headache, and unsteady gait. On admission to hospital, there were marked left cerebellar signs, but no papilloedema. Air ventriculography showed a left cerebellar mass, which was explored by Mr. J. M. Potter on 7 October 1964. The tumour was the size of a golf-ball, soft and brownish-maroon in colour. The visible part was excised or sucked away. Histology showed a typical medulloblastoma, composed of close-packed cells, with dark, often elongated nuclei, and abundant mitotic figures (Fig. 1). A Torkildsen ventriculocisternostomy was performed.

Between 2 October 1964 and 12 November 1964 the patient received radiation therapy under Dr. Frank Ellis. The whole brain, spinal cord, and cauda equina down to the level of the coccyx received $2,700 \mathrm{r}$ with $300 \mathrm{kV}$ $x$-rays. During this period the total dose to the posterior fossa was built up by means of supplementary fields to $3,500 \mathrm{r}$ by means of cobalt gamma-rays. The earlier symptoms disappeared, and normal life was resumed.

In December 1965 there was a recurrence of morning vomiting, which later became projectile. By the end of January there were attacks of headache and vertigo, followed by diplopia and mistiness of vision, drowsiness, and occasional pain in the legs when bending. On readmission to the neurosurgical ward, there was again gross left-sided ataxia, with nystagmus and left abducens weakness. The occipital decompression was full, but pulsating. On 5 February 1966, 16 months after the first operation, the posterior fossa was re-explored. Tumour was now found in the right cerebellar hemisphere and in the fourth ventricle. The histology of this was identical with that seen after the first operation (Fig. 2).

Between 16 February 1966 and 4 March 1966 a total of $2,340 \mathrm{r}$ was given, with cobalt gamma-rays, to the whole brain, but not to the spinal cord, using lateral opposing $27 \mathrm{~cm} \times 12 \mathrm{~cm}$ fields. The course was not completed, because of deterioration in the patient's condition. The arms, and later the legs, became paralysed, and finally there was respiratory paralysis. Death occurred on 14 March 1966, 10 days after the last dose of irradiation.

At necropsy (R.I. p.m. no. 297/66) there was earlyo N broncho-pneumonia; otherwise, abnormalities were cone fined to the head and spinal canal. There was alopecia

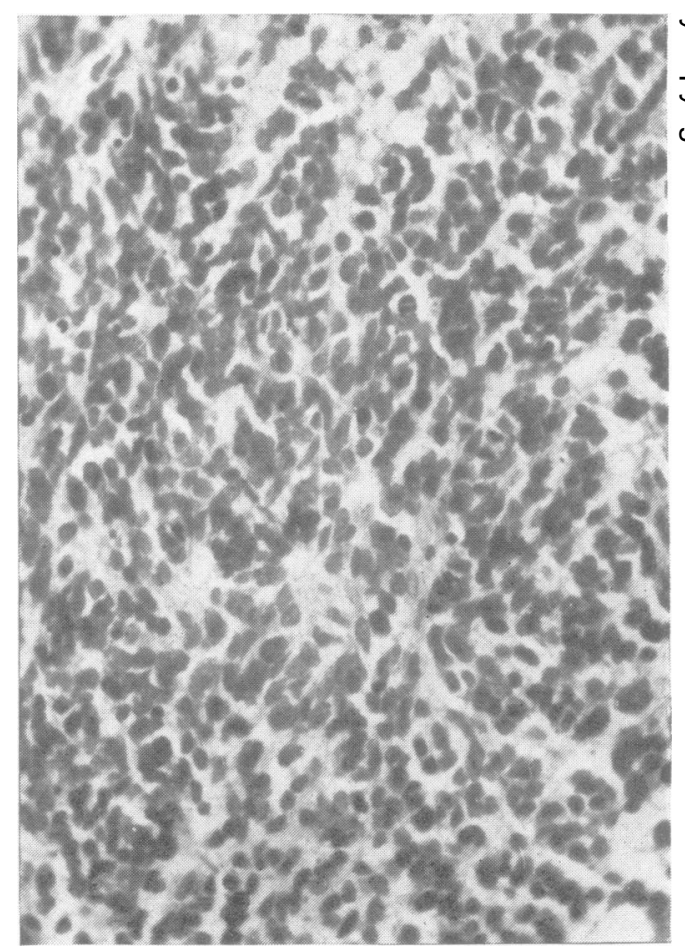

FIG. 1. Tumour from first biopsy. H. \& E. $\times 250$. 


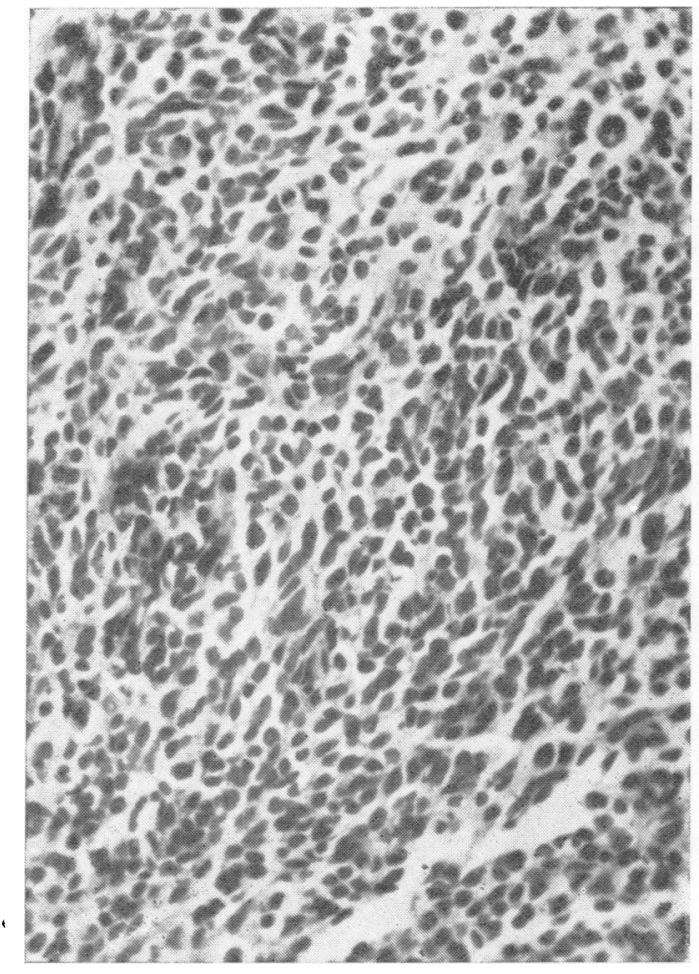

FIG. 2. Tumour from second biopsy. H. \& E. $\times 250$.

from radiation, of the back of the head. The occipital wounds were healthy, and there was no large accumulation of fluid in the decompression. The Torkildsen tube was patent.

The brain $(1,625 \mathrm{~g})$ was swollen, with flattened convolutions. The leptomeninges were opaque in many areas, and frank tumour deposits were found on the under surfaces of both frontal and temporal lobes, hypothalamus, and brain-stem, in the Sylvian fissures, and on the upper surface of the left cerebellar hemisphere, attached to the tentorium. Beneath this was a mass, $3 \mathrm{~cm}$ in diameter, of tumour and scar tissue. There was surgical damage in the vermis. The spinal subarachnoid space was completely obliterated by tumour, and in places it was hard to define the margin between tumour and spinal cord.

Microscopically, the tumour enveloping the spinal cord was indistinguishable from the first and second biopsy specimens, except that a few small areas of necrosis were present (Figs. 3 and 4). There was superficial invasion of the cord, partly by erosion, partly by perivascular infiltration, characteristic of medulloblastoma. Nerve roots, though completely invested in tumour, were only rarely invaded. There was extensive infiltration of both pia and arachnoid, and clumps of tumour cells were present on both sides of the spinal dura. As before, mitotic figures were very numerous.

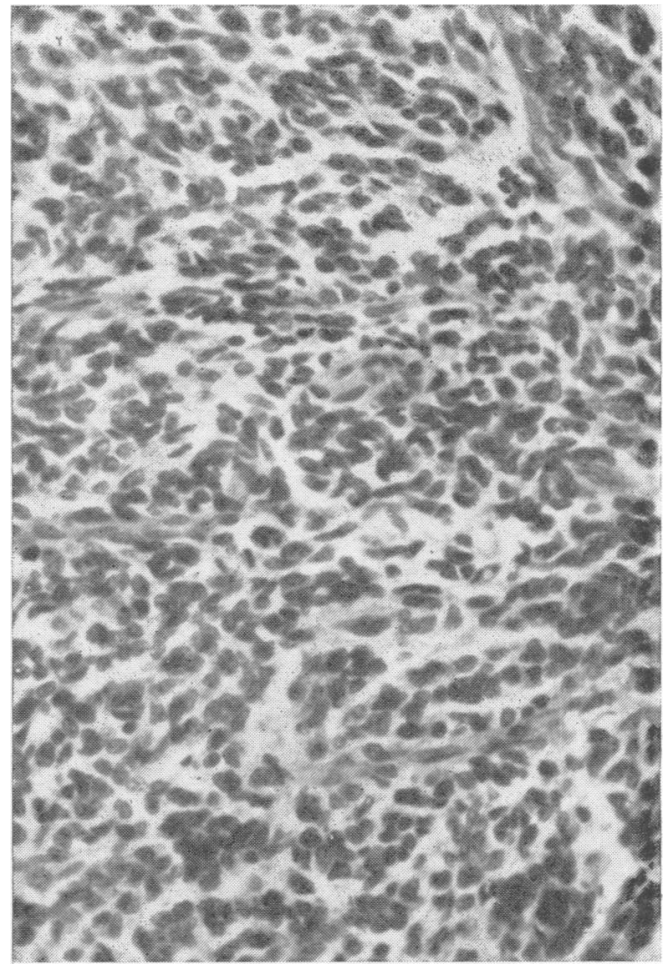

FIC. 3. Tumour from spinal subarachnoid space. H. \& E. $\times 250$.

In all parts of the brain, including the primary site in the cerebellum, the histological appearance of the tumour was utterly different from this. From the lower end of the medulla upwards, there was no tissue which could be recognized as medulloblastoma. Instead, the tumour was highly pleomorphic, being composed of irregular cells of all shapes and sizes, with numerous bizarre giant cells having giant or multiple nuclei. Many of them showed karyorrhexis, and were filled with a coarse chromatin dust (Figs. 5 and 6). In places the tumour was frankly necrotic, and contained numerous swollen phagocytic cells; but the tumour vessels, of all sizes, appeared healthy, and there was no sign of thrombosis. The pattern of surface invasion and perivascular infiltration was the same as that seen in the spinal cord. The nervous parenchyma and its blood vessels were unremarkable, and showed none of the changes seen in radiation encephalopathy.

\section{COMMENT}

There can be little doubt in this case that the histological change in the intracranial parts of the spreading tumour was due to the final course of radiotherapy. The cerebellar biopsy taken $5 \frac{1}{2}$ weeks before death had shown a typical medullo- 


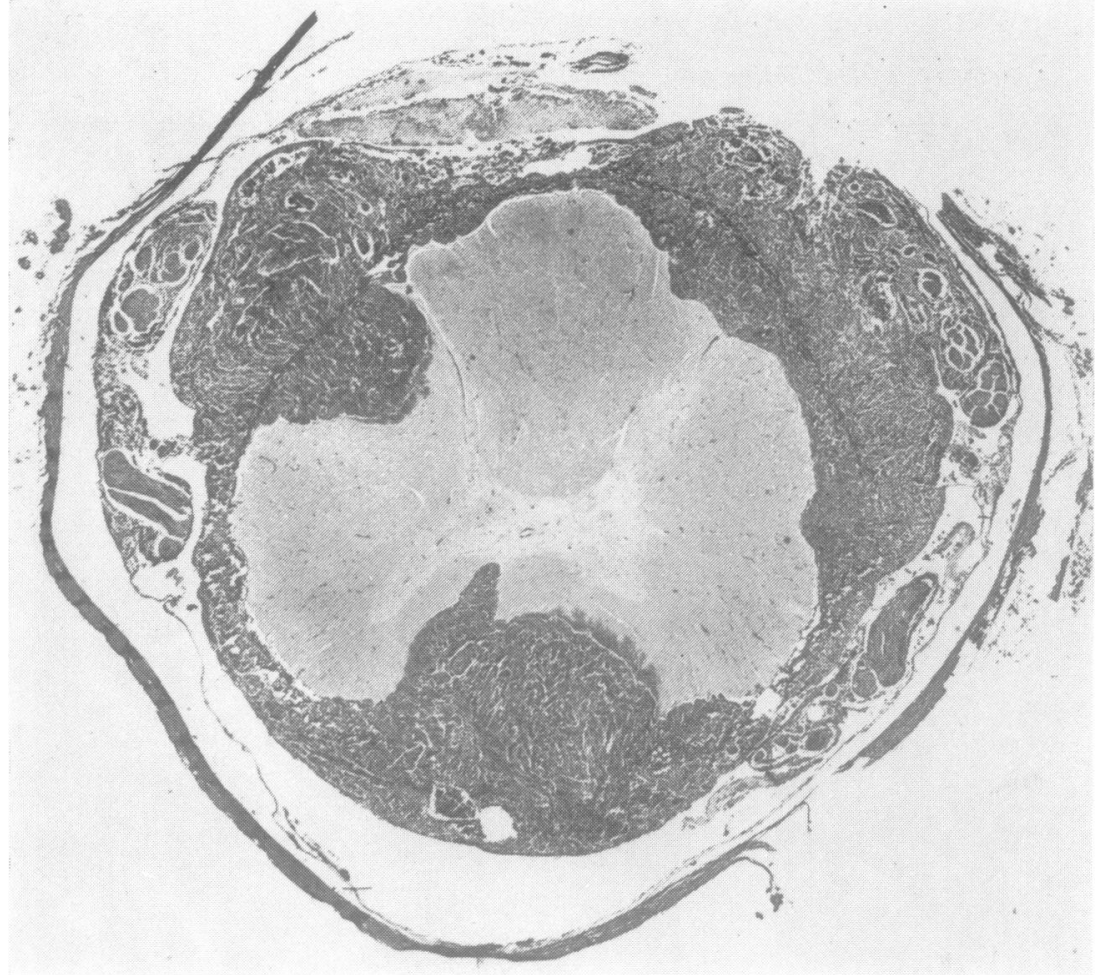

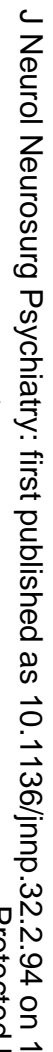

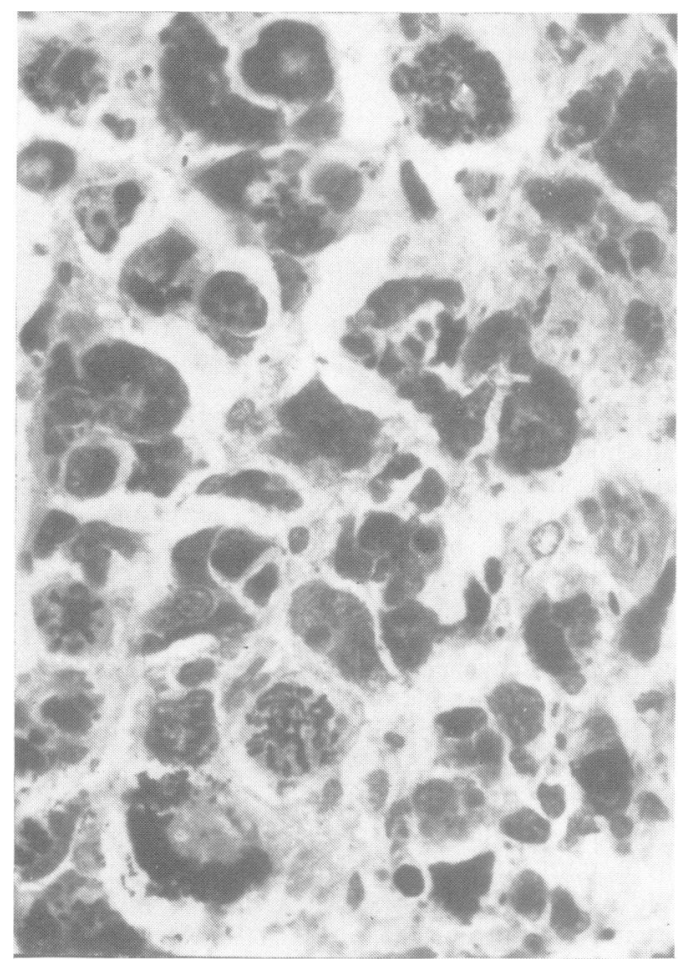

FIG. 5. Tumour in cerebellum. Monstrous cells, and figures of karyorrhexis. H. \& E. $\times 250$.

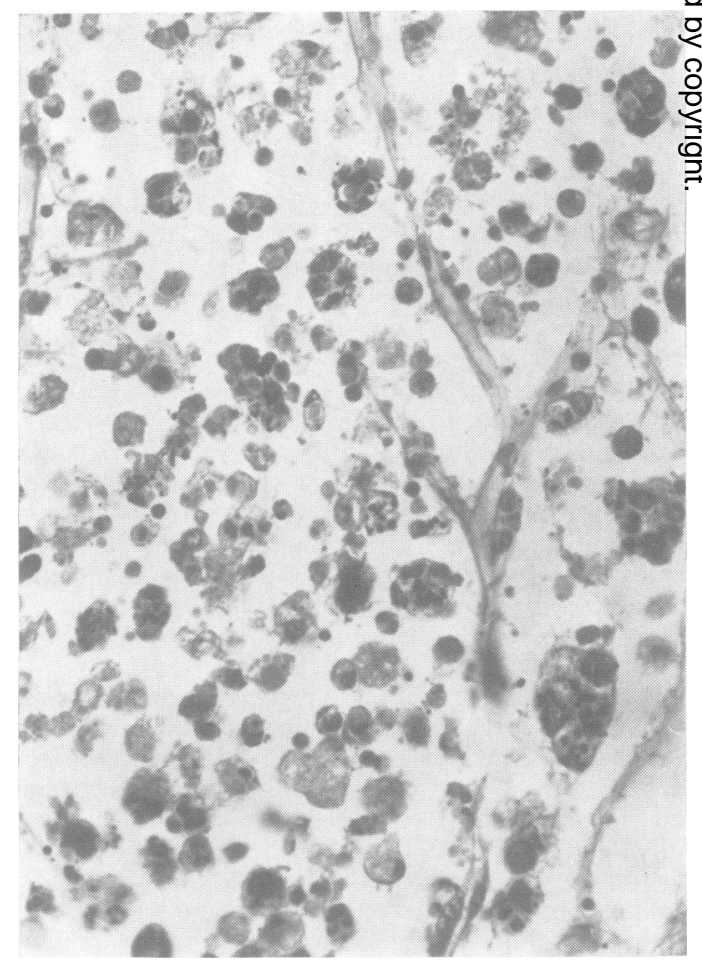

FIG. 6. Tumour in pineal region, with necrosis and numerous phagocytes. $H . \& E . \times 250$.

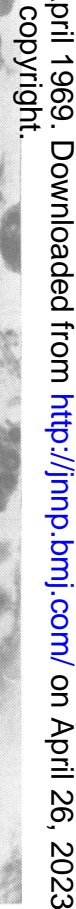

FIG. 4. Thoracic cord

H. \& v.G. $\times 7$.

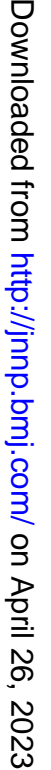


blastoma, and identical tumour was found at necropsy around the spinal cord; but the intracranial parts of the tumour at the time of death had a completely different appearance, characterized by widespread necrosis and cellular pleomorphism, and closely resembling a certain type of giant-celled glioblastoma. There was only one point at which the two types of tumour tissue were seen side by side, namely at the junction of the medulla and the spinal cord (Fig. 7). The other point of interest is that the spinal parts of the tumour, which showed no pleomorphism, had been irradiated at an earlier date. A possible explanation of this would be that the pleomorphic tumour is incapable of reproduction. If we suppose that the initial course of radiotherapy had evoked a pleomorphic change, we may also suspect that the 'typical' tissue found at necropsy was derived from cells which had escaped this radiation effect.

Bailey (1930) gave a brief description of a similar change in a typical medulloblastoma, as seen in a

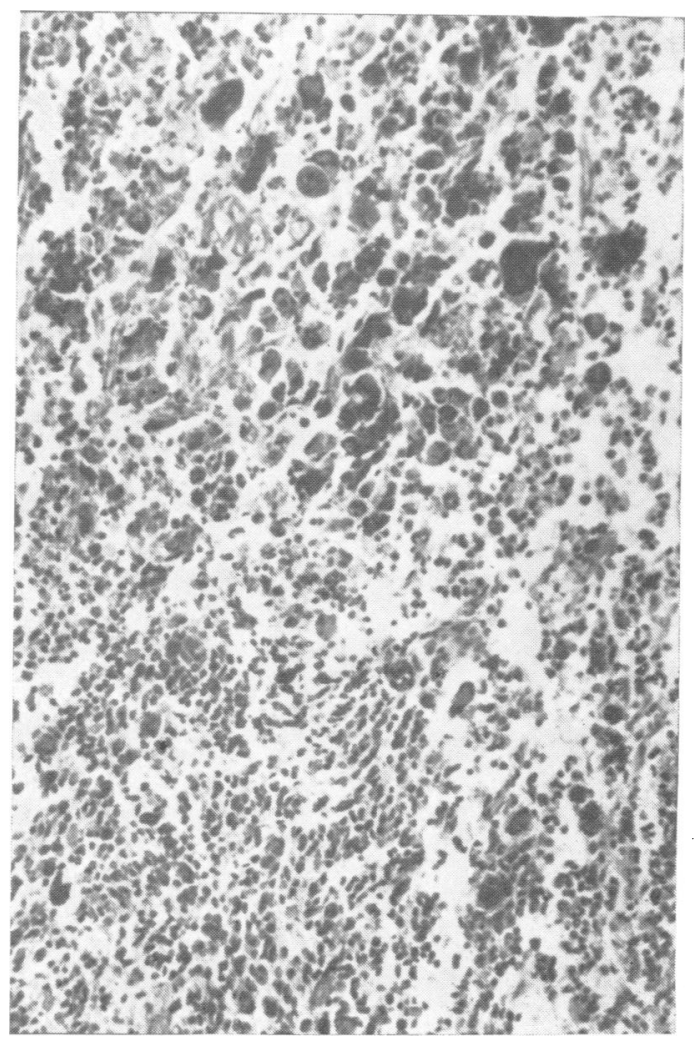

FIG. 7. Tumour in subarachnoid space at spinomedullary junction. Above: pleomorphic tumour. Below: medulloblastoma. H. \& E. $\times 100$. small biopsy specimen removed at re-exploration following a course of radiotherapy, which had been spread over the previous five months. The last dose had been given 11 days before the operation. Bailey speaks of innumerable giant cells containing a dozen or more nuclei. . . Mitoses were frequent, but rarely normal'. By 'mitoses', he presumably refers to figures of karyorrhexis, which have sometimes been called 'pseudomitoses'. I have not come across other reports of this appearance in treated medulloblastomas; but a very similar change is reported by Edelman, Aleu, Scheinberg, Evans, and Davidoff (1964) following irradiation of implanted cerebral gliomas in mice. Two days after irradiation they observed hyperchromasia, pyknosis, and karyorrhexis in tumour cells. A few days later, 'Some cells assume giant proportions. Their nuclei become large and hyperchromatic, and have an irregular outline and a grotesque appearance. Multinucleated giant tumour cells are seen occasionally. Regardless of this atypical pleomorphism, the neoplastic potential of these elements appears questionable. They are seen exclusively in tumours undergoing involution, and probably represent reactive forms to $x$-ray injury in a degenerating phase'. At 12 days, in these animals, tumour necrosis is almost complete.

These authors report a high proportion of apparent 'cures' in mice treated with doses of 2,200 to $3,000 \mathrm{~V}$. They are probably right in regarding the pleomorphic transformation as a stage in the extirpation of the tumour. If so, and if one may argue from the mouse tumour to the human one, it may well be that in the case described here a curative dose of radiation had been delivered to the cranial part of the tumour. In spite of the repeated disappointments of the past 40 years, there are still grounds for hoping that radiotherapy will eventually achieve success in this particularly hateful disease (Lampe and MacIntyre, 1954).

\section{SUMMARY}

An 11-year-old boy, with medulloblastomatosis of the central nervous system, died 10 days after a course of radiotherapy directed to the head only. The tumour surrounding and invading the spinal cord was a typical medulloblastoma, identical with specimens taken from the cerebellum before irradiation. The tumour in and around the brain had a completely different appearance, resembling that of a giant-celled glioblastoma. Much of the latter tissue was necrotic; the rest was probably degenerate and non-viable. The change is almost certainly the result of irradiation. 
Thanks are due to Mr. J. M. Potter and Dr. Hugh Ellis for permission to use their case notes; to Dr. Frank Ellis and Professor Dorothy Russell for advice and help; and to Dr. C. H. Paine for supplying the details of the patient's radiotherapy.

\section{REFERENCES}

Alpers, B. J., and Pancoast, H. K. (1933). The effect of irradiation on normal and neoplastic brain tissue. Amer. J. Cancer, 17, 7-24.

Bailey, P. (1930). Further notes on the cerebellar medulloblastomas; the effect of roentgen radiation Amer. J. Path., 6, 125-136.
- Sosman, M. C., and van Dessel, A. (1928). Roentgen therapy of gliomas of the brain. Amer. J. Roentgenol., 19, 203-264.

Edelman, F. L. Aleu, F. Scheinberg, L. C., Evans, J. C., and Davidoff, L. M. (1964). Effect of irradiation on the growth of intracerebral gliomas in mice. J. Neuropath. exp. Neurol., 23, $1-17$.

Frazier, C. H., Alpers, B. J., Pendergrass, E. P., and Chamberlin G. W. (1937). The effects of irradiation on gliomas. Amer.J. Roentgenol., 38, 203-237.

Lampe, I., and MacIntyre, R. S. (1954). Experiences in the radiation therapy of medulloblastoma of the cerebellum. Amer. J. Roentgenol., 71, 659-668.

Tarlov, I. M. (1937). Effect of roentgenotherapy on gliomas. Arch. Neurol. Psychiat. (Chic.), 38, 513-536. 